\title{
Deficiency in the autophagy modulator Dram1 exacerbates pyroptotic cell death of Mycobacteria- infected macrophages
}

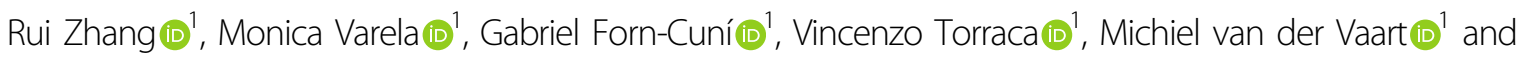 \\ Annemarie H. Meijer (i) ${ }^{1}$
}

\begin{abstract}
DNA damage regulated autophagy modulator 1 (DRAM1) is a stress-inducible regulator of autophagy and cell death. DRAM1 has been implicated in cancer, myocardial infarction, and infectious diseases, but the molecular and cellular functions of this transmembrane protein remain poorly understood. Previously, we have proposed DRAM1 as a host resistance factor for tuberculosis (TB) and a potential target for host-directed anti-infective therapies. In this study, we generated a zebrafish dram 1 mutant and investigated its loss-of-function effects during Mycobacterium marinum (Mm) infection, a widely used model in TB research. In agreement with previous knockdown analysis, dram 1 mutation increased the susceptibility of zebrafish larvae to Mm infection. RNA sequencing revealed major effects of Dram1 deficiency on metabolic, immune response, and cell death pathways during Mm infection, and only minor effects on proteinase and metabolic pathways were found under uninfected conditions. Furthermore, unchallenged dram 1 mutants did not display overt autophagic defects, but autophagic targeting of $\mathrm{Mm}$ was reduced in the absence of Dram1. The phagocytic ability of macrophages in dram 1 mutants was unaffected, but acidification of Mm-containing vesicles was strongly reduced, indicating that Dram1 is required for phagosome maturation. By in vivo imaging, we observed that Dram1-deficient macrophages fail to restrict Mm during early stages of infection. The resulting increase in bacterial burden could be reverted by knockdown of inflammatory caspase a (caspa) and gasdermin Eb (gsdmeb), demonstrating pyroptosis as the mechanism underlying premature cell death of $\mathrm{Mm}$-infected macrophages in dram 1 mutants. Collectively, these data demonstrate that dissemination of mycobacterial infection in zebrafish larvae is promoted in the absence of Dram1 due to reduced maturation of mycobacteria-containing vesicles, failed intracellular containment, and consequent pyroptotic death of infected macrophages. These results provide new evidence that Dram1 plays a central role in host resistance to intracellular infection, acting at the crossroad of autophagy and cell death.
\end{abstract}

\section{Introduction}

Autophagy is an intracellular degradation mechanism that functions to maintain homeostasis and intersects with the initiation of cell death programs when

\footnotetext{
Correspondence: Annemarie H. Meijer (a.h.meijer@biology.leidenuniv.nl) ${ }^{1}$ Institute of Biology Leiden, Leiden University, Einsteinweg 55, 2333 CC Leiden, The Netherlands

These authors contributed equally: Rui Zhang, Monica Varela

These authors jointly supervised this work: Michiel van der Vaart, Annemarie H. Meijer
}

Edited by B. Zhivotovsky homeostasis is perturbed ${ }^{1,2}$. Autophagy can be induced by various stressors, such as nutrient deprivation and UV damage, but also infection. Detection of microbial invaders by the innate immune system activates the autophagy machinery to capture intracellular pathogens in doublemembrane autophagosomes and target them to lysosomal degradation ${ }^{3}$. Autophagy proteins can also be recruited to single-membrane compartments when pathogens are engulfed by phagocytic cells ${ }^{3}$. These autophagic defense mechanisms form promising targets for the development

\section{(c) The Author(s) 2020}

(c) Open Access This article is licensed under a Creative Commons Attribution 4.0 International License, which permits use, sharing, adaptation, distribution and reproduction cc) in any medium or format, as long as you give appropriate credit to the original author(s) and the source, provide a link to the Creative Commons license, and indicate if changes were made. The images or other third party material in this article are included in the article's Creative Commons license, unless indicated otherwise in a credit line to the material. If material is not included in the article's Creative Commons license and your intended use is not permitted by statutory regulation or exceeds the permitted use, you will need to obtain permission directly from the copyright holder. To view a copy of this license, visit http://creativecommons.org/licenses/by/4.0/. 
of novel host-directed therapies for infectious diseases, many of which are currently complicated by the increasing occurrence of antibiotic resistance ${ }^{4,5}$. This is especially true for tuberculosis (TB), the most lethal infectious disease worldwide. The causative agents of human TB or TBlike disease in poikilothermic animals, Mycobacterium tuberculosis (Mtb) and Mycobacterium marinum (Mm), are widely studied to increase understanding of the role of autophagy in host defense $e^{3,6-10}$.

DNA damage regulated autophagy modulator 1 (DRAM1) is a stress-inducible regulator of autophagy and cell death. DRAM1 and other members of the DRAM family have been linked to cancer, myocardial infarction, HIV infection, and TB, but their molecular and cellular functions remain poorly understood ${ }^{6,11-16}$. Among the five DRAM family members, human DRAM1 was first identified as a p53-induced protein that localizes predominantly to lysosomes and promotes autophagic flux as well as UV-damage-induced apoptosis ${ }^{11}$. In response to mycobacterial infection, DRAM1 transcription is induced by nuclear factor kappa $B(N F k B)$, a central hub in the signaling network regulating the immune system ${ }^{6}$. DRAM1 colocalizes with Mtb in infected human macrophages and is required for host resistance of zebrafish larvae against $\mathrm{Mm}$ infection ${ }^{6}$. Mtb and Mm share the RD1 virulence locus, required for ESX1-dependent escape from phagosomes into the cytosol ${ }^{17}$. Selective autophagy, dependent on ubiquitin receptors, such as p62, may counteract this pathogenic mechanism by delaying the escape process or sequestering cytosolic bacteria ${ }^{3,8}$. In addition, selective autophagy has been shown to deliver anti-microbial ubiquitinated peptides to bacteriacontaining compartments ${ }^{18}$. We have recently shown that selective autophagy receptors are required to control $\mathrm{Mm}$ infection in zebrafish and that the host-protective function of Dram1 requires p62 in this infection model $^{6,19}$.

Cytosolic escape of mycobacteria may accelerate the initiation of host cell death programs ${ }^{8}$. In TB, the death of an infected macrophage triggers its phagocytosis by other macrophages that subsequently also undergo cell death, resulting in a cascade of cell death events and the formation of inflammatory infection foci, called granulomas $^{20}$. The fate of individual infected macrophages is therefore a major determinant of whether granulomas can contain the infection or facilitate dissemination of the infection. It has been previously shown that mycobacteriainfected macrophages can undergo several types of regulated cell death, like apoptosis, necroptosis, and pyroptosis, resulting in different infection outcomes ${ }^{21}$. Apoptosis of infected cells is generally regarded as a hostprotective defense mechanism against mycobacterial infection, and virulent Mtb therefore actively inhibit apoptosis $^{20,22,23}$. In contrast, necroptosis and pyroptosis are lytic forms of cell death that create an inflammatory environment that may facilitate extracellular growth and disease progression ${ }^{24,25}$.

While our previous work demonstrated a role for Dram1 in autophagic defense against mycobacterial infection, its potential implication in the regulation of cell death during TB pathogenesis has not been explored. In this study, we generated a dram1 mutant zebrafish line to address this question. Analysis of the mutant fish showed that Dram1 is required for maturation of Mm-containing vesicles and for macrophages to restrict $\mathrm{Mm}$ infection. Without functional Dram1, Mm-infected macrophages prematurely die via a mechanism dependent on inflammatory Caspase a (Caspa) and Gasdermin eb (Gsmdeb) activities, indicative of pyroptosis. Collectively, our data support that Dram1 protects against mycobacterial infection by modulating autophagic targeting and maturation of Mm-containing vesicles. In the absence of Dram1, infected macrophages rapidly become overburdened by the bacteria and initiate pyroptotic cell death, resulting in increased dissemination of the infection.

\section{Results \\ dram 1 null mutants display increased susceptibility to mycobacterial infection}

To study the host resistance function of Dram1, we generated a zebrafish mutant line using CRISPR/Cas9 technology (Fig. S1a). The selected $\operatorname{dram} 1^{\Delta 19 n / \Delta 19 n}$ allele (designated dram $^{i b l 53}$ ) contains a 21 nucleotides deletion combined with a two nucleotides insertion in the first coding exon (Figs. 1a and S1b), which results in undetectable levels of Dram1 protein, supporting that this represents a null allele (Fig. 1b). The mutant was outcrossed to transgenic lines with an autophagy reporter $\mathrm{Tg}$ $(C M V: G F P-m a p 1 l c 3 b)^{26}$ or a macrophage marker $\mathrm{Tg}$ (mpeg1:mCherryF ${ }^{27}$, hereafter referred to as GFP-Lc3 and mpeg1:mCherry. The offspring from incrossed heterozygous fish $\left(\operatorname{dram} 1^{+/ \Delta 19 n}\right)$ strictly followed Mendelian inheritance (Fig. S1d, e), and homozygous mutants were fertile. Body size measurements indicated no apparent difference in development between $\operatorname{dram} 1^{\Delta 19 n / \Delta 19 n}$ and dram $1^{+/+}$larvae (Fig. S1c). Furthermore, the terminal deoxynucleotidyl transferase dUTP nick end labeling (TUNEL) assay did not reveal an effect of dram1 mutation on the basal level of cell death in zebrafish larvae (Fig. 1c, d). In the absence of detectable developmental aberrations, we proceeded to investigate the response of dram1 mutants to $\mathrm{Mm}$ infection. Consistent with previous knockdown results ${ }^{6}, \operatorname{dram}^{\Delta 19 n / \Delta 19 n}$ larvae showed significantly increased susceptibility to infection (Fig. 1e, f). Furthermore, Dram1-deficient larvae displayed accumulation of bacteria inside intersegmental blood vessels, indicative of extracellular bacterial growth (Fig. 1e). 


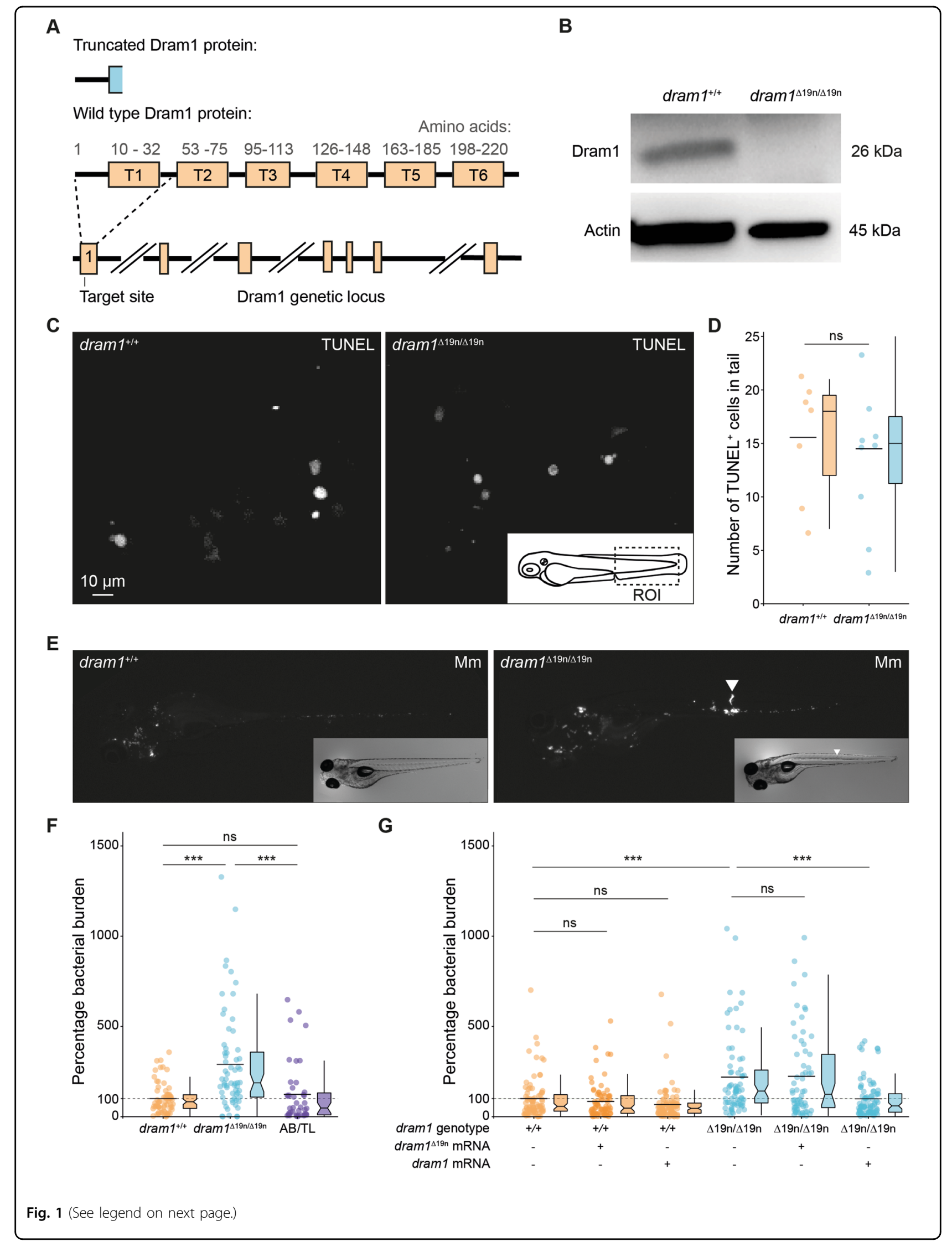


(see figure on previous page)

Fig. 1 Dram 1 deficiency leads to increased susceptibility to $\mathbf{M m}$ infection. a Schematic representation of the zebrafish dram1/Dram1 (ENSDARG00000045561/ENSDARP0 0000066996.3) genetic and protein domain architecture and CRISPR/Cas9 target site. Dram1 (240 amino acids) contains six transmembrane domains (indicated with boxes and labeled T1-T6 with amino acid numbers indicated above). The gene is depicted with coding exons as boxes and introns as solid black lines (introns not drawn to scale). The position of the CRISPR/Cas9 target site and the predicted truncated protein is indicated. $\mathbf{b}$ Confirmation of dram 1 mutation by Western blotting analysis. Protein samples were extracted from $4 \mathrm{dpf} d r a m 1^{\Delta 19 n / \Delta 19 n}$ and $d r a m 1^{+/+}$larvae (>10 larvae/sample). The blots were probed with antibodies against Dram1 and Actin as a loading control. c Representative confocal micrographs of sections from the tail region showing TUNEL staining performed on dram $1^{\Delta 19 n / \Delta 19 n}$ and dram $1^{+/+}$larvae at $3 \mathrm{dpf}$. Scale bar, $10 \mu \mathrm{m}$. d Quantification of TUNEL-positive cells in the indicated region of the tail of dram $1^{\Delta 19 \mathrm{n} / \Delta 19 \mathrm{n}}$ and $\mathrm{dram} 1^{+/+}$larvae at $3 \mathrm{dpf}(\geq 7 \mathrm{larvae} / \mathrm{group})$. Data are represented by scatter and boxplots as detailed in the "Methods" section. e Representative stereo images of infected dram $1^{\Delta 19 n / \Delta 19 n}$ and dram $1^{+/+}$larvae at $3 \mathrm{dpi}$. The arrowhead indicates the accumulation of bacteria in intersegmental veins. $\mathbf{f}$ and $\mathbf{g}$ Quantification of bacterial burdens at 3 dpi for dram 1 mutants, wild type siblings, and unrelated wild types $\mathbf{f}$ or for $d r a m 1$ and $d r a m 1^{\Delta 19 n}$ mRNA injected individuals $\mathbf{g}$. The data are accumulated from two independent experiments ( $>42$ larvae/group for $\mathbf{f}$ and $>62$ larvae/group for $\mathbf{g}$ ) and represented by scatter and boxplots as detailed in the methods section.

We detected no differences in bacterial burden between dram $1^{+/+}$and unrelated wild types, indicating that the genetic background did not affect its susceptibility to infection (Fig. 1f). Next, we demonstrated that injection of dram 1 mRNA could rescue the infection susceptibility phenotype of $d r a m 1^{\Delta 19 n / \Delta 19 n}$, while injection of a control mRNA containing the $\Delta 19 \mathrm{n}$ deletion could not (Fig. 1g). Collectively, our analysis of $\operatorname{dram} 1^{\Delta 19 n / \Delta 19 n}$ zebrafish larvae confirms that Dram1 is necessary for host defense during $\mathrm{Mm}$ infection.

\section{Dram1 deficiency affects transcriptional regulation of metabolic, immune response, and cell death pathways during mycobacterial infection}

To further explore the $d r a m 1^{\Delta 19 n / \Delta 19 n}$ phenotype, we performed RNA sequencing. We aimed to identify changes in the gene expression network caused by the absence of functional Dram1, rather than by the increased level of infection in mutants. Therefore, $\operatorname{dram} 1^{\Delta 19 \mathrm{n} / \Delta 19 \mathrm{n}}$ larvae were infected with two different dosages of bacteria. A dosage of $300 \mathrm{CFU}$, resulting in a higher bacterial burden compared with $\mathrm{rram}^{+/+}$, and a lower dosage of $150 \mathrm{CFU}$ resulting in a similar bacterial burden as in $\operatorname{dram} 1^{+/+}$ infected with $300 \mathrm{CFU}$ (Figs. 2a and S2a). The analysis time point, 4 days post-infection (dpi), correlates with mycobacterial granuloma formation and strong transcriptional activation of the immune response ${ }^{28}$. Principal component analysis showed clear differences between Mm-infected larvae and PBS-injected controls and between the $\operatorname{dram} 1^{\Delta 19 \mathrm{n} / \Delta 19 \mathrm{n}}$ and $d \mathrm{ram} 1^{+/+}$groups (Fig. $\mathrm{S} 2 \mathrm{~b})$. Differential gene expression analysis comparing PBS-injected dram1 $1^{\Delta 19 n / \Delta 19 n}$ and dram $1^{+/+}$groups showed that Dram1 deficiency influences the gene regulation network to a relatively small extent under noninfected conditions (Fig. S2c). Gene ontology and gene set-enrichment analysis (GSEA) revealed that these relatively minor transcriptome differences in non-infected dram1 mutants were related to changes in metabolic and proteolytic pathways (Table S1). During Mm infection, we found that infected wild type larvae displayed a pronounced level of differential gene expression (both upregulated and downregulated genes) compared to their PBS-injected wild type controls, consistent with previous results $^{28}$. In constrast, alterations in the gene regulation network were less pronounced when comparing infected $\operatorname{dram} 1^{\Delta 19 \mathrm{n} / \Delta 19 \mathrm{n}}$ mutants to their PBS-injected mutant controls, irrespective of whether they where infected with 150 or $300 \mathrm{CFU}$ (Fig. S2c).

While differences between $d r a m 1^{\Delta 19 n / \Delta 19 n}$ mutants and controls were observed at both infection doses, the transcriptome response of mutants infected with $300 \mathrm{CFU}$ showed more overlap with infected controls than mutants infected with $150 \mathrm{CFU}$, despite their higher bacterial burden. For example, while expression of genes related to host defense pathways, such as Nod-like receptor (NLR) signaling, phagosome-related processes, cytokine signaling, and apoptosis, were commonly affected in all Mminfected larvae, other immune-related pathways, like Tolllike receptor (TLR) and RIG-I-like receptors signaling, were not affected in $\operatorname{dram}^{\Delta 19 \mathrm{n} / \Delta 19 \mathrm{n}}$ larvae infected with $150 \mathrm{CFU}$ Mm (Fig. 2b). This difference may reflect a milder initial immune response towards infection with only half the inoculum compared to the other two groups. Despite this, $\sim 60 \%$ of the infection-responsive genes in dram1 $1^{+/+}$(1170 out of 1971) were not differentially expressed in $\operatorname{dram}^{\Delta 19 \mathrm{n} / \Delta 19 \mathrm{n}}$ mutants (Fig. S2d). Alteration of metabolic pathways related to energy and carbon metabolism (e.g. glycolysis, TCA cycle), a characteristic of mycobacterial infections ${ }^{29}$, was markedly absent in $\operatorname{dram} 1^{\Delta 19 \mathrm{n} / \Delta 19 \mathrm{n}}$ larvae (Fig. 2b). In contrast, infection of $\operatorname{dram} 1^{\Delta 19 \mathrm{n} / \Delta 19 \mathrm{n}}$ with $300 \mathrm{CFU}$ influenced the expression of genes related to other metabolic processes, such as cholesterol and amino acid biosynthesis (Fig. 2b). For further analysis, we visualized the differential expression of genes that are part of relevant Kyoto Encyclopedia of Genes and Genomes (KEGG) pathways for the three Mm infected groups compared to their respective controls. In the KEGG pathway of TLR signaling, the visualization 


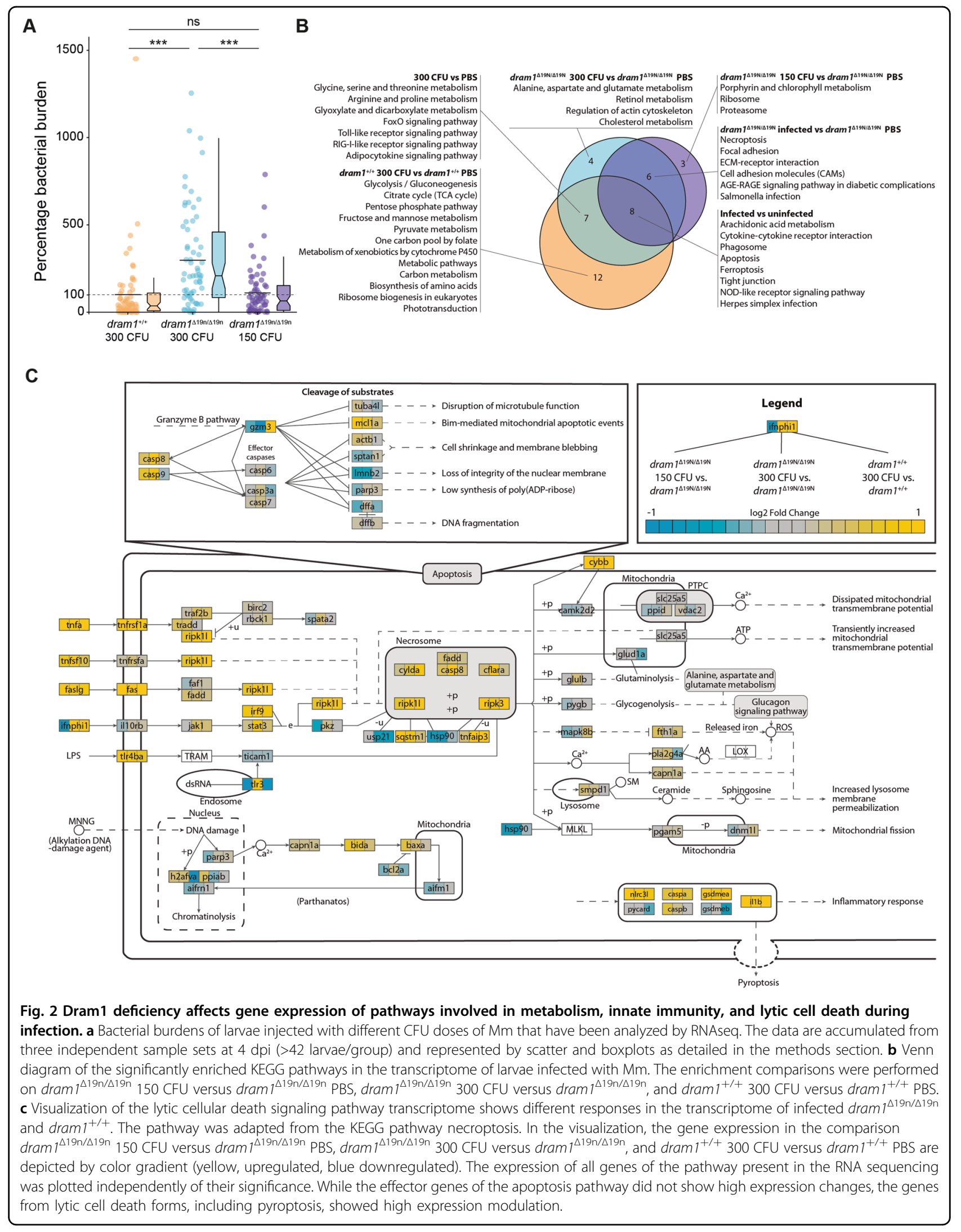


revealed differential expression of several TLRs and downstream genes between the wild type and dram 1 mutant infected groups (Fig. S3). In cell death-related KEGG pathways, Dram1 deficiency affected the regulation of programmed cell death during infection, resulting in enhanced expression of genes involved in lytic forms of cell death (Fig. 2c).

\section{The autophagic response to $\mathrm{Mm}$ infection is altered in dram 1 mutants}

Altered metabolic pathway regulation in dram1 mutants might be a compensatory response to defects in autophagy. Investigating GFP-Lc3 puncta as a marker for autophagosome formation revealed no difference between $\operatorname{dram} 1^{\Delta 19 \mathrm{n} / \Delta 19 \mathrm{n}}$ and $d \mathrm{ram} 1^{+/+}$larvae under unchallenged conditions (Fig. 3a). However, $\operatorname{dram1}^{\Delta 19 \mathrm{n} / \Delta 19 \mathrm{n}}$ larvae responded differently by accumulating higher levels of GFP-Lc3 and Lc3 incorporated in autophagosomes (Lc3II) compared with $\operatorname{dram} 1^{+/+}$when we applied a cellular stress factor, Bafilomycin A1 (BafA1), which inhibits vacuolar $\mathrm{H}+\mathrm{ATPase}$ (V-ATPase) to prevent autophagolysosomal maturation (Fig. 3b, c). In agreement, protein levels of ubiquitin-binding receptors, p62 and Optineurin, which are substrates of autophagy ${ }^{30}$, were unaffected in unchallenged $d r a m 1^{\Delta 19 n / \Delta 19 n}$ larvae but were elevated compared to the levels in $\operatorname{dram}^{+/+}$following BafA1 treatment (Fig. 3d). Similar to BafA1 treatment, Mm infection-induced Lc3-II to higher levels in $\operatorname{dram1}^{\Delta 19 \mathrm{n} /}$ ${ }^{\Delta 19 n}$ than in $\operatorname{dram1}^{+/+}$(Fig. 3e). However, colocalization analysis between GFP-Lc3 and Mm showed that $\operatorname{dram1}^{\Delta 19 \mathrm{n} / \Delta 19 \mathrm{n}}$ larvae displayed significantly less GFPLc3-positive Mm clusters compared to $\mathrm{dram}^{+/+}$(Fig. 3f, $\mathrm{g}$ ), indicating that autophagic targeting of $\mathrm{Mm}$ is reduced in the absence of Dram1, despite an overall increase in Lc3-II accumulation. Taken together, dram 1 mutants display no overt autophagic defects in unchallenged conditions, but are affected in their autophagic response to cellular stress, including intracellular infection by $\mathrm{Mm}$.

\section{Dram1 deficiency does not affect phagocytosis of $\mathrm{Mm}$}

We wanted to exclude that reduced GFP-Lc3 association with $\mathrm{Mm}$ might be a consequence of a defect in phagocytosis by macrophages, the primary niche for intracellular $\mathrm{Mm}$ growth ${ }^{31}$. First, we verified that Dram1 deficiency did not alter the total number of macrophages, labeled by mpeg1:mCherryF (Fig. 4a). Similarly, there was no effect on the other main innate immune cell population of zebrafish larvae, the neutrophils (Fig. 4b). Next, we assessed phagocytic activity at $1 \mathrm{~h}$ after intravenous delivery of $\mathrm{Mm}$. The results showed that $\mathrm{Mm}$ was phagocytosed by macrophages in $\operatorname{dram} 1^{\Delta 19 n / \Delta 19 n}$ and dram $1^{+/+}$at a similar rate, as indicated by the percentage of intracellular Mm (Fig. 4c). We then determined at which time point during the infection a difference in bacterial burden between $\operatorname{dram} 1^{\Delta 19 n / \Delta 19 n}$ and $d r a m 1^{+/+}$ was first detectable. We found that Dram1 deficiency significantly increased $\mathrm{Mm}$ infection burden at 2 dpi but not yet at $1 \mathrm{dpi}$ (Fig. 5a). In conclusion, both $\operatorname{dram}^{119 n / \Delta 19 n}$ and $d r a m 1^{+/+}$can phagocytose the injected dose of $\mathrm{Mm}$ within the first hour after infection, and the immunocompromised state of Dram1-deficient larvae first becomes apparent 2 days later.

\section{Dram1 is required for macrophages to restrict $\mathrm{Mm}$ infection}

Since Dram1 protein localizes to lysosomes ${ }^{11}$, we asked whether Dram1-deficiency affected the maturation of Mm-containing vesicles. We used LysoTracker to determine the extent of colocalization between $\mathrm{Mm}$ and acidic vesicles at an early stage of infection ( $1 \mathrm{dpi})$, before differences in bacterial burden were detectable. Approximately $60 \%$ of $\mathrm{Mm}$ clusters were LysoTrackerpositive in $\operatorname{dram} 1^{+/+}$, while this was reduced to $20 \%$ in $\operatorname{dram1}^{\Delta 19 n / \Delta 19 n}$ (Fig. 5b, c). Next, we asked whether reduced maturation of $\mathrm{Mm}$-containing vesicles limits the ability of macrophages in $\operatorname{dram} 1^{\Delta 19 n / \Delta 19 n}$ hosts to combat the infection. We found that at $1 \mathrm{dpi}$ the majority of $\mathrm{Mm}$ clusters were restricted inside macrophages for both $\operatorname{dram} 1^{\Delta 19 n / \Delta 19 n}$ and $d r a m 1^{+/+}$hosts (Fig. 5d, e). However, at $2 \mathrm{dpi}$, the majority of $\mathrm{Mm}(65 \%)$ resided inside macrophages in $d r a m 1^{+/+}$, while we observed an increased escape of Mm from macrophages in $\operatorname{dram} 1^{\Delta 19 n / \Delta 19 n}$ larvae, with only $31 \%$ remaining intracellular (Fig. 5f, g). Furthermore, we frequently observed remnants of dead macrophages in the proximity of bacterial clusters in $\operatorname{dram} 1^{\Delta 19 n / \Delta 19 n}$ (Fig. 5f), which most likely were contained within the macrophage prior to its death. Together, these data demonstrate that Dram1 is necessary for macrophages to contain the infection and prevent extracellular bacterial growth.

\section{Dram 1 deficiency results in increased pyroptotic cell death of $\mathrm{Mm}$-infected macrophages}

Transcriptome analysis had revealed that Dram1 deficiency affects lytic cell death pathways, including necroptosis and pyroptosis, while effects on the apoptosis pathway were relatively minor. To delineate the mechanism responsible for increased cell death of $\mathrm{Mm}$ infected macrophages in dram 1 mutants, we performed TUNEL staining, which detects damaged DNA present both in apoptotic and pyroptotic cells ${ }^{32,33}$. We observed TUNEL-positive cells around $\mathrm{Mm}$ clusters both in $\operatorname{dram} 1^{\Delta 19 n / \Delta 19 n}$ and $d r a m 1^{+/+}$, but the frequency was around 2.1 times higher in the mutants (Fig. 6a, b). We observed no difference in activation (cleavage) of Caspase 3 , an important executioner of apoptosis ${ }^{34,35}$, between $\operatorname{dram} 1^{\Delta 19 n / \Delta 19 n}$ and $d r a m 1^{+/+}$in the absence or presence of $\mathrm{Mm}$ infection (Fig. 6c). Therefore, we asked if the 

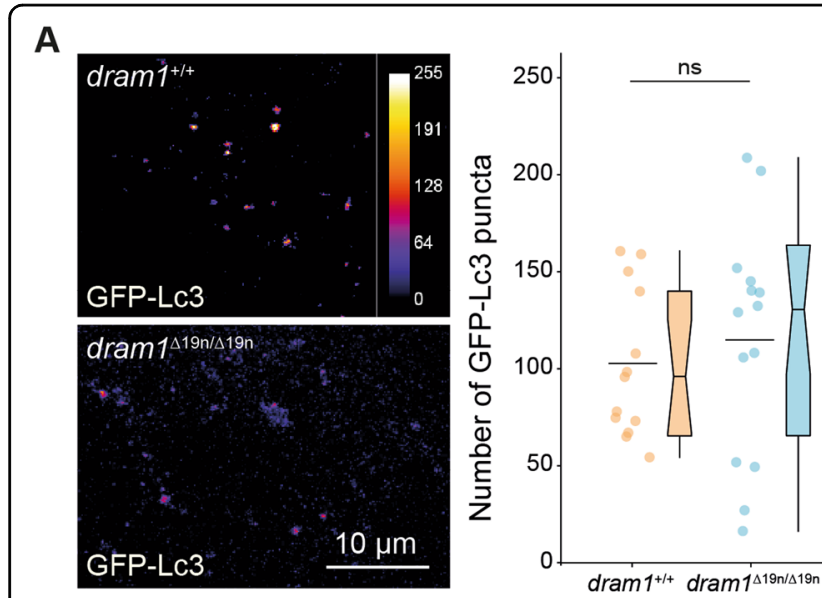

C

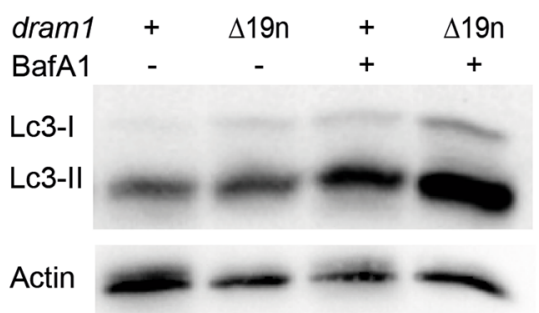

$16 \mathrm{kDa}$

$14 \mathrm{kDa}$

$45 \mathrm{kDa}$

D

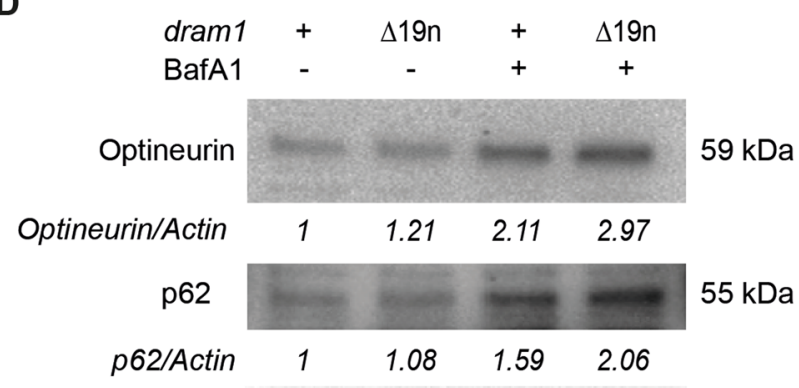

Actin

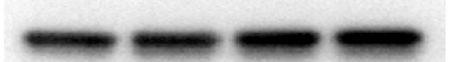

$45 \mathrm{kDa}$

E

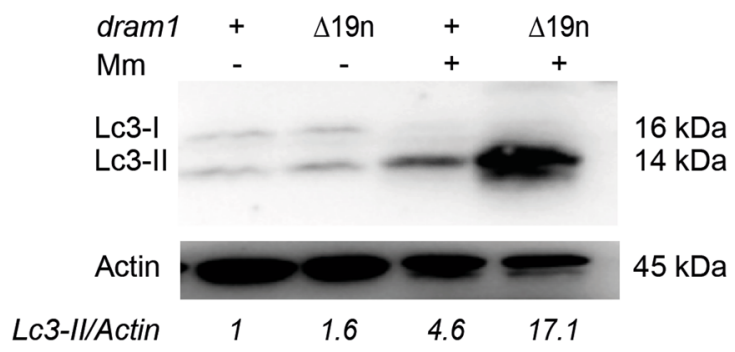

B
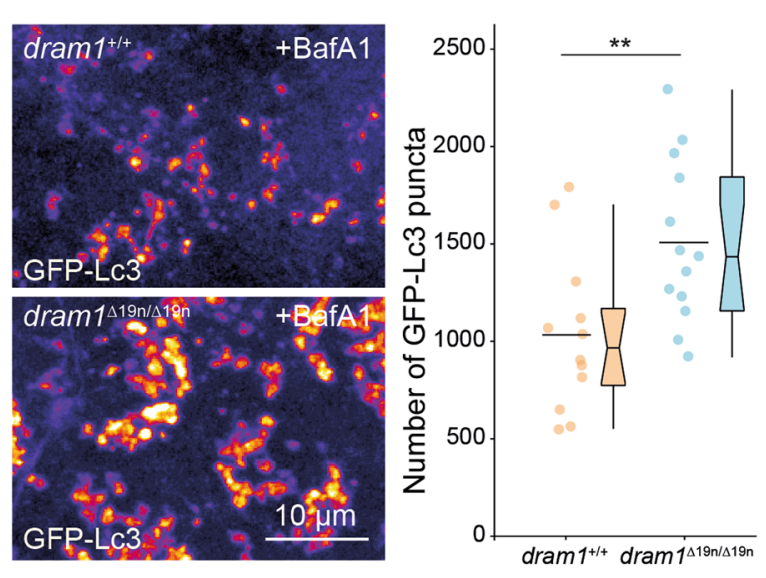

$\mathbf{F}$
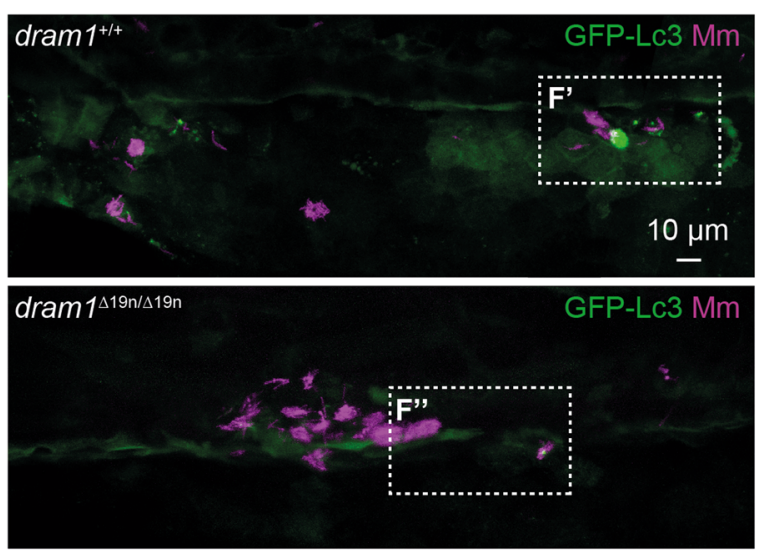

G

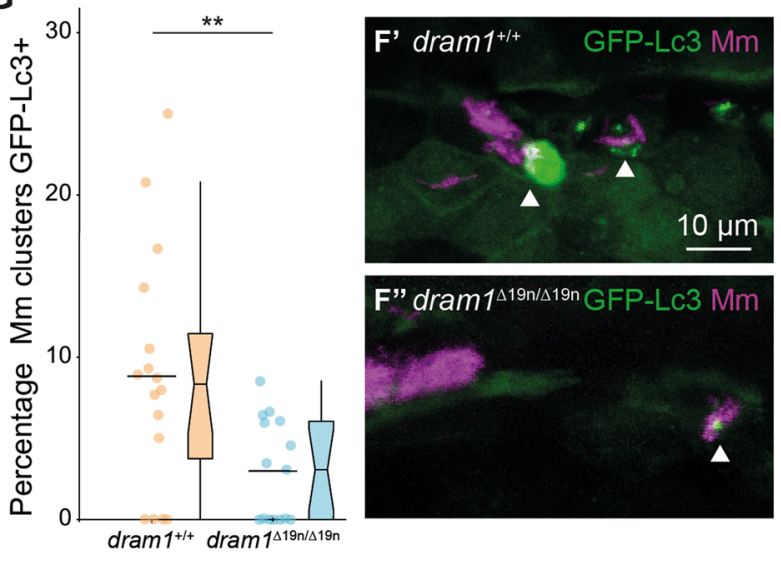

Fig. 3 (See legend on next page.)

increased cell death of $\mathrm{Mm}$-infected macrophages $\operatorname{dram} 1^{\Delta 19 n / \Delta 19 n}$ was due to pyroptosis. Pyroptotic cell death is associated with the activity of inflammatory caspases, like Caspase 1 and Caspase 4/5/11, and is characterized by the formation of Gasdermin pores in the cell membrane ${ }^{36-38}$. We have recently found that Caspase a (Caspa) is the Caspase-family member that induces pyroptosis of $\mathrm{Mm}$-infected macrophages in zebrafish via 
(see figure on previous page)

Fig. 3 Dram1 is required for GFP-Lc3 targeting to Mm clusters. $\mathbf{a}$, b Representative confocal micrographs and quantification of GFP-LC3 puncta in $d r a m 1^{\Delta 19 n / \Delta 19 n}$ and $d r a m 1^{+/+}$larvae in an unstimulated situation (basal autophagy, a) and following BafA1 treatment $\mathbf{b}$. Each larva was imaged at a pre-defined region of the tail fin ( $\geq 11$ larvae/group). Results are accumulated from two independent experiments and represented by scatter and boxplots as detailed in the "Methods" section. ns non-significant, ${ }^{*} p<0.05,{ }^{* *} p<0.01$, ${ }^{* * *} p<0.001$. Scale bars, $10 \mu m$. The intensity calibration bar for the Lookup table (LUT) is displayed in panel a. c-e Western blot analysis of autophagy. Protein samples were obtained from $4 \mathrm{dpf} d \mathrm{dram} 7^{\Delta 19 \mathrm{n} / \Delta 19 \mathrm{n}}$ and $\mathrm{dram}^{+/+}$larvae (>10 larvae/sample). Lc3 $\mathbf{c}$ and $\mathbf{e}$, or p62 and Optineurin $\mathbf{d}$ protein levels were detected in absence or presence of BafA1, $\mathbf{c}$ and $\mathbf{d}$, or in the presence or absence of $\mathrm{Mm} \mathbf{e}$. Actin was used as a loading control. Western Blots were repeated three, $\mathbf{c}$ and $\mathbf{d}$, or two e times with protein extracts derived from independent experiments. The Lc3ll/Actin or p62/Actin and Optineurin/Actin ratio, normalized to the control sample, is indicated below the blots. $\mathbf{f}-\mathbf{g}$ Representative confocal micrographs and quantification of GFP-LC3 co-localization with Mm clusters in infected $\operatorname{dram} 1^{\Delta 19 n / \Delta 19 n}$ and $d r a m 1^{+/+}$larvae. The top images $\mathbf{f}$ show the entire region of imaging, while the bottom images $\mathbf{f}^{\prime}$ and $\mathbf{f}^{\prime \prime}$ show details of GFP-LC3 colocalization of Mm clusters in dram $1^{\Delta 19 n / \Delta 19 n}$ and dram $1^{+/+}$larvae. The arrowheads indicate GFP-LC3-positive Mm clusters. The data is accumulated from two independent experiments ( $\geq 15$ larvae/group) and represented by scatter and boxplots as detailed in the "Methods" section. Scale bars, $10 \mu \mathrm{m}$.

Gasdermin Eb (Gsdmeb) ${ }^{39,40}$. Thus, we analyzed Caspa activity at $2 \mathrm{dpf}$, the time point where we observed increased cell death in $\operatorname{dram} 1^{\Delta 19 n / \Delta 19 n}$. We detected a minor but significant increase of whole larvae Caspa levels in $\operatorname{dram} 1^{\Delta 19 n / \Delta 19 n}$ infected with Mm compared with the non-infected mutant, but such infection-dependent increase was not observed in $\operatorname{dram1}^{+/+}$(Fig. 6d). Next, we asked if the increased bacterial burden in Dram1deficient larvae is dependent on Caspa activity. Knockdown of caspa reduced bacterial burden in $\operatorname{dram} 1^{+/+}$. Furthermore, caspa knockdown also reduced the enhanced bacterial burden of $\operatorname{dram} 1^{\Delta 19 / \Delta 19}$, bringing the infection burden in mutants and wild types to a comparable low level (Fig. 6e). Similarly, gsdmeb knockdown reduced bacteria burden in $d r a m 1^{+/+}$and rescued the hypersusceptibility phenotype of $\operatorname{dram}^{\Delta 19 / \Delta 19}$ (Fig. 6f). Collectively, these data suggest that dissemination of mycobacterial infection in zebrafish embryos is promoted in the absence of Dram1 due to reduced bacterial containment and consequent pyroptosis of infected macrophages.

\section{Discussion}

The lysosomal protein DRAM1/Dram1 regulates autophagy and cell survival/death decisions under multiple stress conditions, including diseases like cancer and infection. Its mechanism of action remains largely unknown. Here, we have demonstrated that dram1 mutation in zebrafish impairs resistance to mycobacterial infection. We show that Dram1 deficiency reduces autophagic targeting of $\mathrm{Mm}$ and acidification of $\mathrm{Mm}$ containing vesicles, ultimately resulting in pyroptotic cell death of infected macrophages and increased extracellular growth of mycobacteria during early stages of the infection.

The dram1 mutant generated for this study was characterized by transcriptome analysis. Under unchallenged conditions, we found that deficiency of Dram 1 affects the network of gene regulation to a small degree, with detectable differences in proteinase and metabolic pathways. Considering that lysosomes act as central regulatory units in signal transduction, this transcriptional response might be a compensatory mechanism for defects in lysosomal function due to the deficiency in Dram $1^{41}$. The differential expression of metabolic pathway genes was markedly enhanced in response to Mm infection. Recent studies have shown that the metabolic status of macrophages is critical for their innate host defense function ${ }^{42}$. Therefore, the metabolic dysregulation in dram 1 mutants could contribute to their hypersusceptibility to $\mathrm{Mm}$ infection. Altered regulation of essential innate immunity signaling pathways (including TLR signaling) in dram1 mutants could also contribute to this phenotype. Finally, the upregulated expression of genes in lytic cell death pathways may contribute to pathological inflammation and is consistent with the increased bacterial burden in dram1 mutants.

The function of DRAM1 as a modulator of autophagy has been studied well in vitro ${ }^{11}$. We therefore tested whether zebrafish dram1 mutants display defects in autophagic processes. Autophagy is a host response to diverse stress factors, including starvation. Zebrafish larvae until $5 \mathrm{dpf}$ can rely on their yolk proteins for nutrients $^{43}$, and we therefore assumed that their autophagic processes are not activated above a level normal for their developmental stage, unless autophagy is triggered by a stressor such as infection. In agreement, we did not detect any differences when comparing the basal levels of autophagy activity in uninfected dram 1 mutant larvae of 4 dpf to those of their wild type siblings. This finding is consistent with an in vitro study of the function of mouse DRAM1 ${ }^{44}$, which showed that basal autophagy was not altered in the absence of DRAM1 in primary mouse embryonic fibroblasts (MEFs). DRAM1 deficiency did not change autophagy induction in response to starvation or while blocking $\mathrm{mTOR}^{45}$. However, the lack of DRAM1 affected the activation of autophagy in human cells (HeLa and A549) following the induction of cellular stress by 


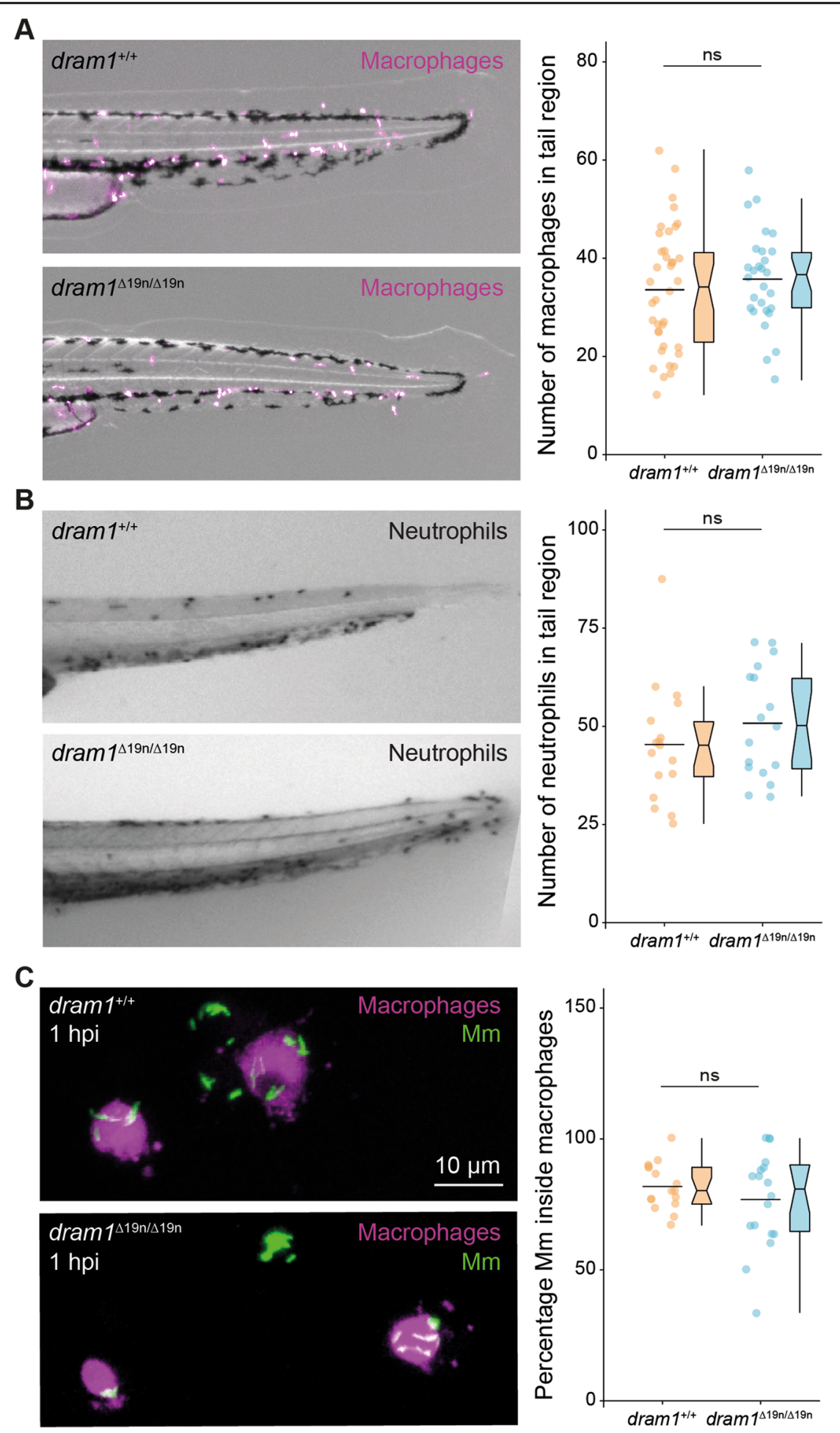

Fig. 4 (See legend on next page.) 
(see figure on previous page)

Fig. 4 Dram 1 deficiency does not affect the capability of macrophages to phagocytose $\mathbf{M m}$. a Representative stereo micrographs of

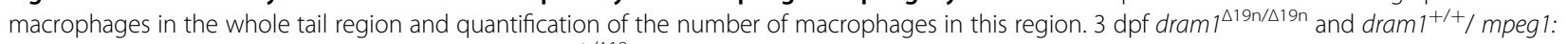
$m C h e r r y F$ larvae were obtained from incrossed dram $1^{+/ \Delta 19 n}$ animals and the number of macrophages for each larva were counted before determining the genotype. Genotyping was performed by PCR and Sanger sequencing ( $\geq 28$ larvae/group). Data are accumulated from two independent experiments and represented by scatter and boxplots as detailed in the "Methods" section. ns non-significant, ${ }^{*} p<0.05$, ${ }^{* *} p<0.01$, ${ }^{* * *} p<0.001$. b Representative stereo images of the whole tail of $3 \mathrm{dpf} d r a m 1^{\Delta 19 n / \Delta 19 n}$ and dram $1^{+/+}$larvae following an immunohistochemical peroxidase activity detection protocol. The number of neutrophils in this region was quantified per individual larva ( $\geq 18$ larvae/group). Data are accumulated from two independent experiments and represented by scatter and boxplots as detailed in the "Methods" section. ns non-significant, ${ }^{*} p<0.05,{ }^{* *} p<0.01,{ }^{* * *} p<0.001$. c Representative confocal micrographs of macrophages located in the blood circulation over the yolk sac of infected dram $1^{\Delta 19 n / \Delta 19 n}$ and dram $1^{+/+}$embryos in mpeg 1:mCherry F background at $1 \mathrm{~h}$ post-infection (hpi). Scale bars, $10 \mu \mathrm{m}$. Quantification of phagocytosis of $\mathrm{Mm}$ by macrophages at $1 \mathrm{hpi}$. dram $1^{\Delta 19 \mathrm{n} / \Delta 19 \mathrm{n}}$ and $d r a m 1^{+/+}$embryos in mpeg 1:mCherry F background were infected with $\mathrm{Mm}$ at $30 \mathrm{hpf}$ and fixed at 1 hpi. The percentage of macrophages having phagocytosed Mm clusters was determined per individual larva ( $\geq 16$ larvae/ group). Results are accumulated from two independent experiments and represented by scatter and boxplots as detailed in the "Methods" section. ns non-significant, ${ }^{*} p<0.05,{ }^{* *} p<0.01,{ }^{* * *} p<0.001$.

treatment with the mitochondria inhibitor 3nitropropionic acid $(3-\mathrm{NP})^{46}$. Besides infection, DNAdamage, and interference with energy metabolism ${ }^{6,11,14}$, it remains to be further investigated which stress factors can activate DRAM1/Dram1 in vitro and in vivo.

Blocking autophagy flux in dram 1 mutant larvae with BafA1 revealed an increase of GFP-Lc3 puncta and Lc3-II protein levels. The dram 1 mutants also accumulated higher Lc3-II protein levels than controls under conditions of $\mathrm{Mm}$ infection. The prolonged stress conditions imposed to zebrafish larvae during BafA1 treatment or infection may induce a compensatory response in dram1 mutants to produce more autophagosomes. Importantly, despite the increased Lc3-II levels in infected dram1 mutants, imaging in GFP-Lc3 transgenic fish revealed that mycobacteria are targeted by autophagic vesicles nearly three-fold less frequently in dram 1 mutants compared to wild type zebrafish larvae. This reduced autophagic targeting of Mm was not due to a different phagocytic ability of zebrafish macrophages. We did, however, find that Dram1 deficiency reduced acidification of Mm-containing vesicles, which was associated with the premature death of infected macrophages. The resulting higher mycobacterial burden of infected zebrafish is in line with increased expansion of extracellularly growing $\mathrm{Mm}^{47}$. While we found that Mm-infected macrophages die more rapidly in the absence of Dram1, human DRAM1 has been shown to trigger lysosomal membrane permeabilization and cell death in HIV-infected $\mathrm{CD}^{+} \mathrm{T}$ cells, thereby lowering viral replication (14). Together, these studies indicate that DRAM1/Dram1 expression levels can have a major impact on cell death processes during infection, with different outcomes depending on the cell type and infectious agent.

DRAM1 was previously shown to mediate apoptosis by blocking the degradation of the pro-apoptotic protein $\mathrm{Bax}^{12}$, but an effect of Dram1 deficiency on apoptosis was not observed in our infection study. Strikingly, we found that Dram1 deficiency leads to increased inflammatory caspase activity and gasdermin-dependent pyroptotic cell death during infection. Previous studies revealed that pyroptosis can be induced by diverse pathogens and forms a critical mechanism to restrict microbial infection ${ }^{48,49}$. In line with this, there is also evidence that mycobacteria inhibit pyroptosis of infected macrophages via diverse mechanisms ${ }^{50}$. However, recent studies found that lytic cell death (pyroptosis and necroptosis) helps mycobacteria to evade host immunity and disseminate the infection ${ }^{25,51}$. Indeed, in the present study we found that pyroptotic cell death promotes the expansion of mycobacteria in Dram1deficient zebrafish hosts. Moreover, genetic inhibition of Caspase a and Gasdermin Eb could rescue the exacerbated bacterial growth in dram1 mutants, confirming that pyroptosis rather than necroptosis is responsible for the hypersusceptibility phenotype. Taken together, the death of infected macrophages is intricately related to TB pathogenesis and can result either in increased dissemination or restriction of the infection in the zebrafish host. The contradicting evidence discussed above concerning the beneficial or detrimental effects of the different modes of cell death suggests that the balance between different cell death modalities plays a crucial role in determining the outcome of the infection and a careful characterization of the specific type of cell death being studied is critical for a better understanding of TB pathogenesis.

In conclusion, restriction of mycobacteria in infected macrophages during the early stages of infection requires functional Dram1. In this work, we have shown that Dram1 is involved in several processes important to defense against intracellular pathogens, potentially providing an intersection between modulation of autophagy, lysosomal function, and regulated cell death. Future studies are required to precisely elucidate the role of the lysosomal protein Dram1/DRAM1 in this network. Using an in vivo model for the early stages of TB disease we have demonstrated the importance of Dram 1 for the elimination of intracellular mycobacteria and the cell fate of infected macrophages. 
A

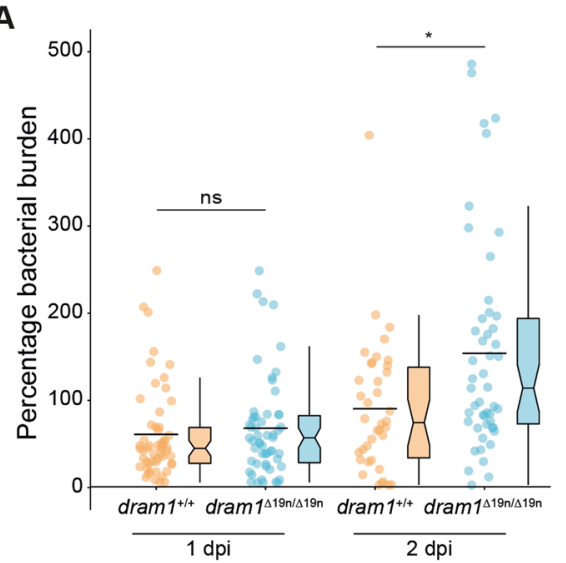

D
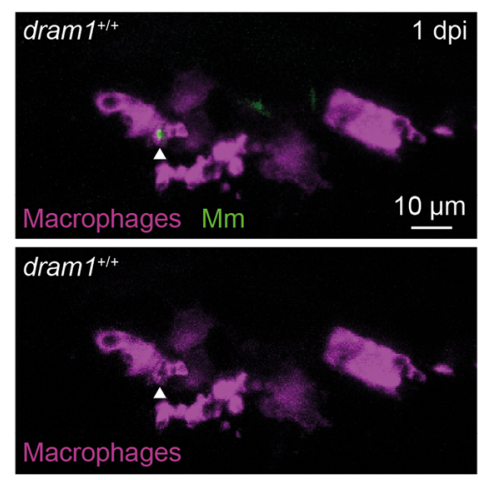

$\mathbf{F}$
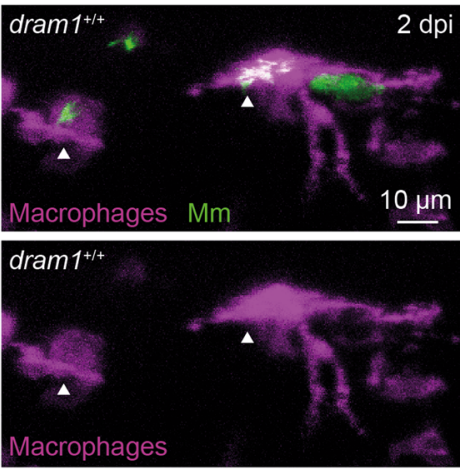

B
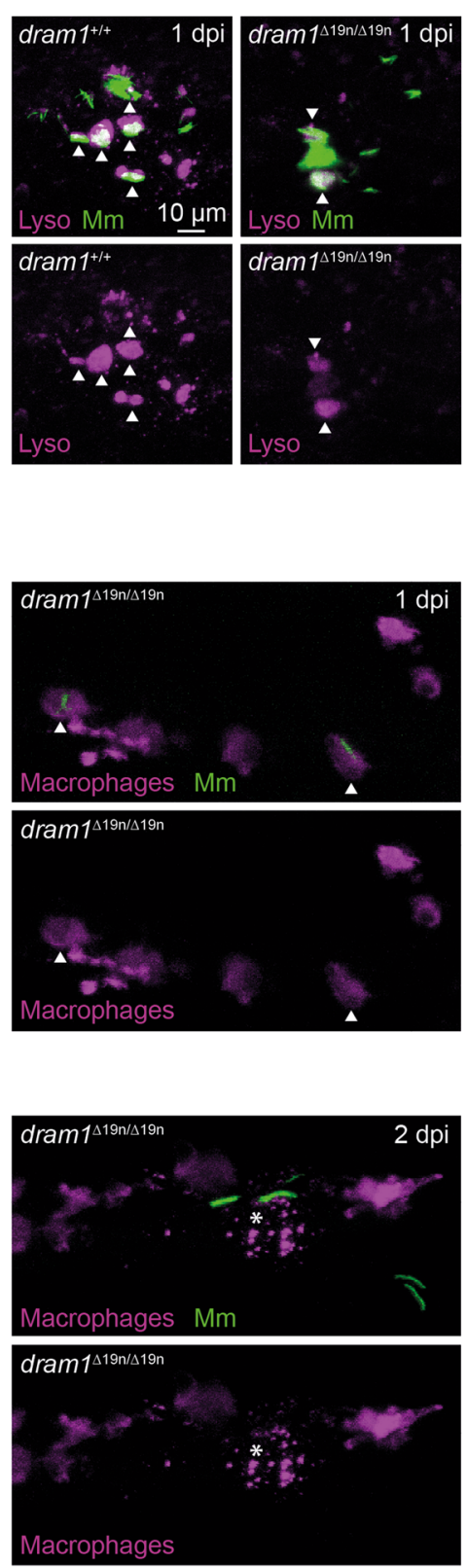

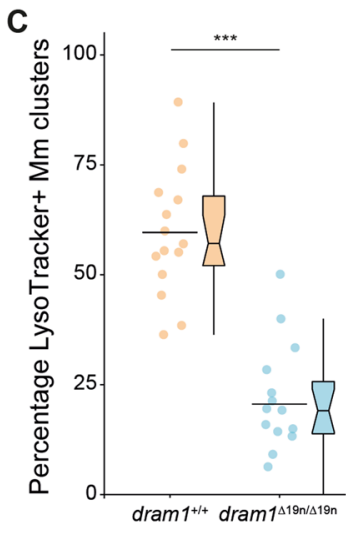

$\mathbf{E}$

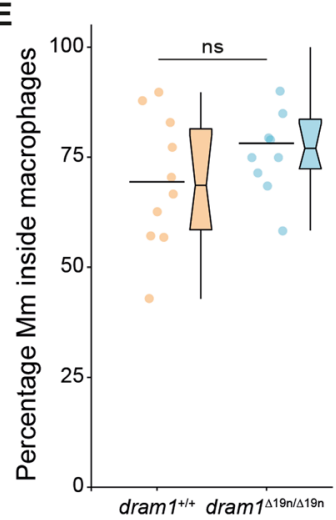

G

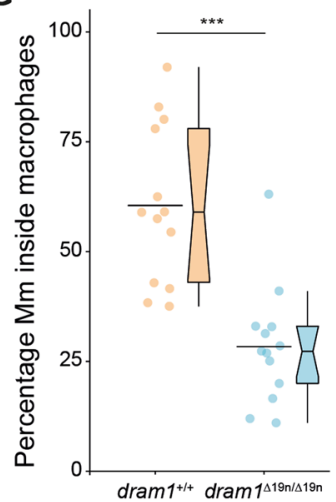

Fig. 5 Macrophages fail to restrict Mm infection in Dram1-deficient larvae. a Mm bacterial burden for $d r a m 1^{\Delta 19 n / \Delta 19 n}$ and $d r a m 1^{+/+}$at 1 and 2 dpi. Data are accumulated from two independent experiments (>38 larvae/group) and represented by scatter and boxplots as detailed in the "Methods" section. b Representative confocal images of LysoTracker staining performed on infected dram $1^{\Delta 19 n / \Delta 19 n}$ and dram $1^{+/+}$embryos at $1 \mathrm{dpi}$. The arrowheads indicate Lysotracker-positive (LysoTracker $\left.{ }^{+}\right)$Mm clusters. Scale bars, $10 \mu \mathrm{m}$. c The percentage of LysoTracker ${ }^{+} \mathrm{Mm}_{\mathrm{m}}$ clusters was determined in infected embryos ( $\geq 15$ embryos/group) at 1 dpi. The percentage of Mm clusters positive for LysoTracker staining (LysoTracker ${ }^{+}$) was determined per individual infected larva. Data are accumulated from two independent experiments and represented by scatter and boxplots as detailed in the "Methods" section. ns non-significant, ${ }^{*} p<0.05,{ }^{* *} p<0.01,{ }^{* * *} p<0.001$. d and $\mathbf{f}$ Representative confocal images of infected dram $1^{\Delta 19 n / \Delta 19 n}$ and dram $1^{+/+}$embryos/larvae in mpeg1:mCherryF background at $1 \mathrm{dpi} \mathbf{d}$ and $2 \mathrm{dpi} \mathbf{f}$. The entire CHT region of fixed embryos or larvae was imaged. The arrowheads indicate intracellular Mm clusters and an asterisk (*) indicates remnants from dead macrophages. Scale bars, $10 \mu \mathrm{m}$. e and $\mathbf{g}$ Percentage of Mm clusters restricted inside macrophages at $1 \mathrm{dpi}$ e and $2 \mathrm{dpi} \mathbf{g}$ ( $\geq 10$ embryos/group). The percentage of intracellular Mm clusters was determined per individual embryo. Data are accumulated from two independent experiments and represented by scatter and boxplots as detailed in the "Methods" section. ns non-significant, ${ }^{*} p<0.05,{ }^{* *} p<0.01,{ }^{* * *} p<0.001$. 
A
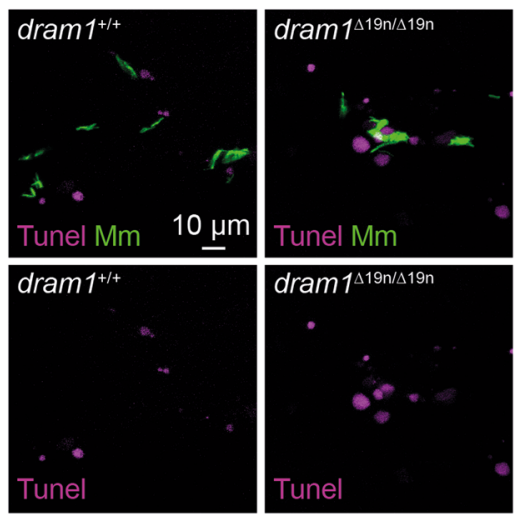

C

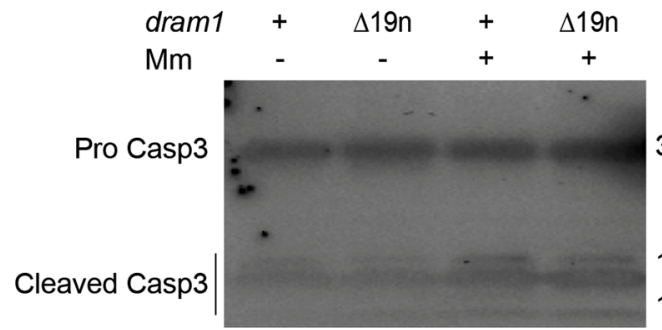

Actin

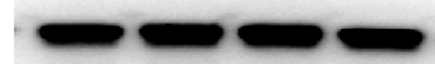

E

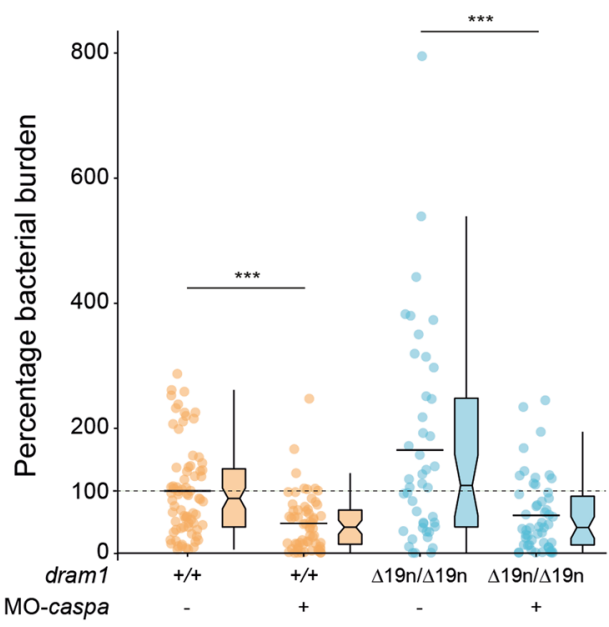

B

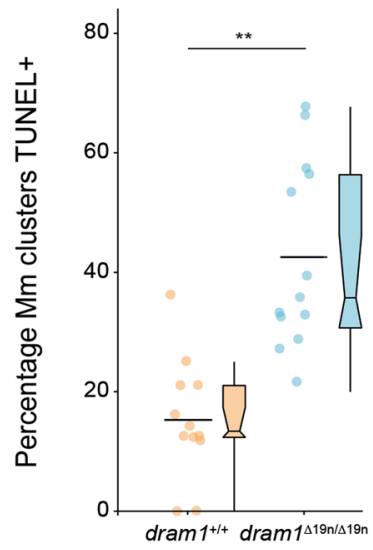

D

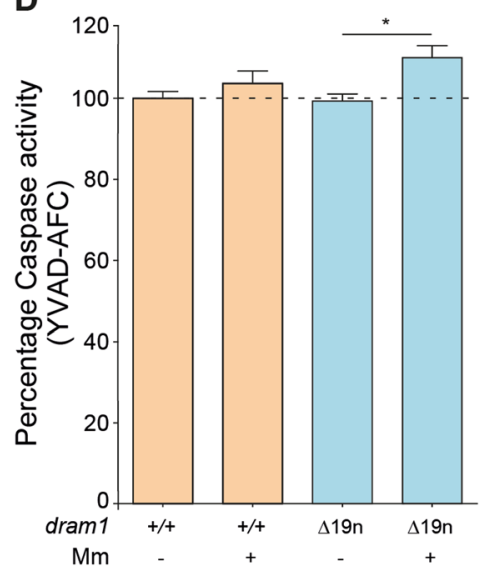

$\mathbf{F}$

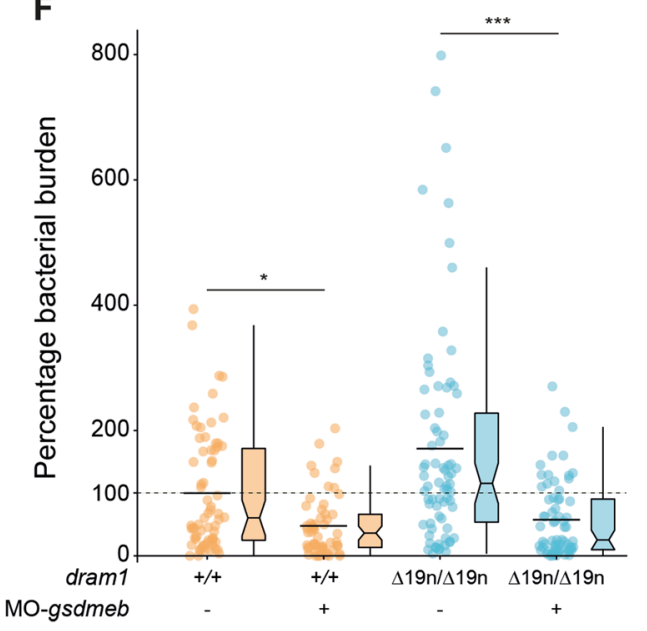

Fig. 6 (See legend on next page.)

Materials and methods

Zebrafish culture and lines

Zebrafish lines in this study (Table S2) were handled in compliance with local animal welfare regulations, as overseen by the Animal Welfare Body of Leiden University (License number: 10612) and maintained according to standard protocols (zfin.org). Generation of the dram 1 mutant was approved by the Animal Experimentation 
(see figure on previous page)

Fig. 6 Dram 1 deficiency results in increased pyroptotic cell death. a Representative confocal images of TUNEL staining in dram $7^{\Delta 19 n / \Delta 19 n}$ and dram $1^{+/+}$larvae at $2 \mathrm{dpi}$. The entire CHT region of fixed $2 \mathrm{dpi} d r a m 1^{\Delta 19 n / \Delta 19 n}$ and dram $1^{+/+}$larvae was imaged. Scale bars, $10 \mu \mathrm{m}$. b Quantification of the percentage of Mm clusters positive for TUNEL staining (TUNEL ${ }^{+}$) in dram $1^{\Delta 19 n / \Delta 19 n}$ and $d r a m 1^{+/+}$larvae. The percentage of Mm clusters TUNEL ${ }^{+}$ in the CHT region was determined per individual infected larva ( $\geq 12$ larvae/group). Data are accumulated from two independent experiments and represented by scatter and boxplots as detailed in the "Methods" section. ns non-significant, ${ }^{*} p<0.05,{ }^{* *} p<0.01,{ }^{* * *} p<0.001$. c Detection of proCaspase 3 and cleaved Caspase 3 protein in $\operatorname{dram}^{\Delta 19 n / \Delta 19 n}$ and $d r a m 1^{+/+}$embryos. Protein samples were extracted from $4 \mathrm{dpf}$ infected and uninfected dram $1^{\Delta 19 n / \Delta 19 n}$ and dram $1^{+/+}$larvae (>10 larvae/sample). The Western blots were probed with antibodies against Caspase 3 and Actin as a loading control. Data is representative of two independent experiments. $\mathbf{d}$ Detection of Caspase activity (YVAD-AFC) in $\operatorname{dram} 7^{\Delta 19 n / \Delta 19 \mathrm{n}}$ and $d r a m 1^{+/+}$ embryos. Protein samples were obtained from $2 \mathrm{dpf}$ control and infected dram $1^{\Delta 19 n / \Delta 19 n}$ and dram $1^{+/+}$embryos in GFP-Lc3 background (35

embryos/sample). The data is accumulated from two independent experiments ( $n=4$ samples/condition). e Mm bacterial burden at $2 \mathrm{dpi}$ following knockdown of caspa in dram $7^{\Delta 19 n / \Delta 19 n}$ and $d r a m l^{+/+}$embryos. The data is accumulated from two independent experiments ( $>44$ larvae/group) and represented by scatter and box plots as detailed in the "Methods" section. ns non-significant, ${ }^{*} p<0.05,{ }^{* *} p<0.01,{ }^{* * *} p<0.001$. f Mm bacterial burden at $2 \mathrm{dpi}$ following knockdown of gsmdeb in $d r a m 1^{\Delta 19 n / \Delta 19 n}$ and $d r a m 1^{+/+}$embryos. The data is accumulated from two independent experiments ( $>56$ larvae/group) and represented by scatter and box plots as detailed in the "Methods" section. ns non-significant, ${ }^{*} p<0.05$, ${ }^{* *} p<0.01$, ${ }^{* * *} p<0.001$ to mutated dram 1 .

Committee of Leiden University (UDEC) under protocol 14198. All experiments were done on embryos or larvae up to 5 days post-fertilization, which have not yet reached the free-feeding stage. Embryos/larvae were kept in egg water $(60 \mu \mathrm{g} / \mathrm{ml}$ Instant Ocean sea salts) containing $0.003 \% 1$ phenyl-2-thiourea (PTU, Sigma-Aldrich) at $28.5^{\circ} \mathrm{C}$ and treated with $0.02 \%$ ethyl 3-aminobenzoate methanesulfonate (Tricaine, Sigma-Aldrich) for anesthesia before bacterial injections, imaging and fixation.

\section{CRISPR/Cas9-mediated mutagenesis of zebrafish dram 1}

Short guide RNAs (sgRNAs) targeting the first coding exon of zebrafish dram1 (ENSDARG00000045561) were designed using the chop-chop website ${ }^{52}$ and generated by PCR complementation and amplification of full-length ssDNA oligonucleotides (Sigma-Aldrich, Table S3) as described $^{19}$. For in vitro transcription of sgRNAs, $0.2 \mu \mathrm{g}$ template DNA was used to generate sgRNAs using the MEGA short script ${ }^{\circ}$ T7 kit (AM1354, ThermoFisher) and purified by RNeasy Mini Elute Clean up kit (74204, Qiagen). The Cas9 mRNA was transcribed using mMESSAGE mMACHINE ${ }^{\circ}$ SP6 Transcription Kit (AM1340, ThermoFisher) from a Cas9 plasmid (39312, Addgene) and purified with RNeasy Mini Elute Clean up kit (74204, Qiagen). A mixture of sgRNA and Cas9 mRNA was injected into one-cell stage $\mathrm{AB} / \mathrm{TL}$ embryos (sgRNA $150 \mathrm{pg} /$ embryo and Cas 9 mRNA $300 \mathrm{pg} /$ embryo). The effect of CRISPR injection was confirmed by PCR and Sanger sequencing. Genotyping was performed by PCRamplification of the genomic region of interest using the following primers: Forward: 5'-AGTGAACGTCC GTGTCTTTCTT-3', Reverse: 5'-ACATCTTGTCGATACAAAGCGA-3'; followed by Sanger sequencing to identify mutations (Base Clear, Netherlands) ${ }^{19}$.

\section{RNA sequencing}

Total RNA was extracted from $5 \mathrm{dpf}$ infected and uninfected snap-frozen larvae (20 larvae/sample) from three independent crosses using Qiazol reagent (79306,
QIAGEN) according to the manufacturer's instructions and extracted with RNeasy Mini kit (74104, QIAGEN). RNAs were quantified using a 2100 Bioanalyzer (Agilent, US). At least 10 million reads per sample were sequenced using Illumina Single read $50 \mathrm{nt}$ runs in a Hiseq2500. Sequencing, mapping the reads against the $D$. rerio GRCz10.80 reference genome and read counting were performed by ZF-screens (Leiden, Netherlands). Analysis of the count libraries was performed in RStudio $1.1 .383^{53}$ running R $3.4 .3^{54}$ using inhouse scripts (available at github.com/gabrifc). An initial quality check of the samples was performed using the tools provided in the edgeR package v3.20.7 ${ }^{55}$. Differential gene expression was assessed via pairwise comparisons using DESeq2 v1.18.1 ${ }^{56}$. Genes with a FDR-adjusted p-value (adjpval) $<0.05$ were considered statistically significant. Venn Diagrams were created using the R package VennDiagram v1.6.18 ${ }^{57}$. Gene lists were ranked using the published function "- $\log _{10}$ (adjpval)" $\log _{2}$ (fold-change)", compared to the $\mathrm{C} 2$ "Curated Gene Sets" collection from the Molecular Signatures Database (MSigDB) using GSEA v3. $0^{58}$. Gene ontology enrichment was analyzed with goseq v1.3.0 $0^{59}$. Updated gene length and Gene Ontology data from the Zv10 assembly was retrieved from Ensembl with the packages ensembldb v2.2.1 ${ }^{60}$ and biomaRt v2.34.2 $2^{61}$, respectively. When necessary, mapping between different database gene identifiers was also performed using biomaRt v2.34.2. KEGG pathway analysis was performed with the kegga function provided in limma v3.34.5 ${ }^{62}$. Gene regulation data of significant pathways was visualized with pathview v1.18.063. Raw data are deposited into Gene Expression Omnibus under accession number GSE129035.

\section{Western blot analysis}

Western blot analysis was performed as previously described $^{19}$. Antibodies used were as follows: polyclonal rabbit anti-DRAM1 (N-terminal) (1:1000, ARP47432-P050, Aviva systems biology), polyclonal rabbit anti-Optineurin (C-terminal) (1:200, lot\#100000; Cayman Chemical), polyclonal rabbit anti-p62 (C-terminal) (1:1000, PM045, 
lot\#019, MBL), polyclonal rabbit anti-Lc3 (1:1000, NB100-2331, lot\#AB-3, Novus Biologicals), monoclonal Caspase 3 antibody (1:1000, \#9662, Lot\#12, Cell Signaling), anti-mono-and polyubiquitinated conjugates mouse monoclonal antibody (1:200; BML-PW8810-0100, lot $\# 01031445$, Enzo life Sciences), polyclonal actin antibody (1:1000, 4968S, lot\#3, Cell Signaling), anti-rabbit IgG, HRP-Linked Antibody (1:1000, 7074 S, Lot\#0026, Cell Signaling), anti-mouse IgG, HRP-linked antibody (1:3000, 7076S, Lot\#029, Cell Signaling).

\section{Infection conditions and bacterial burden quantification}

$\mathrm{Mm}$ strain $\mathrm{M}$ or $\mathrm{Mm}$ strain 20 fluorescently labeled with mWasabi or mCherry, respectively ${ }^{64,65}$, were microinjected into the blood island of embryos at $28 \mathrm{hpf}$ as previously described $^{66}$. The injection dose was $200 \mathrm{CFU}$ for all experiments, except for the phagocytosis assay (500 CFU), and the RNA sequencing of infected wild types (300 CFU) and dram 1 mutants (150 and $300 \mathrm{CFU}$ ). Embryos were manually dechorionated by tweezers before the injection. Infected embryos were imaged using a Leica MZ16FA stereo fluorescence microscope equipped with a DFC420C color camera, and the number of bacterial pixels per infected fish were obtained from the individual embryo stereo fluorescence images using previously described software ${ }^{67}$.

\section{Confocal laser scanning microscopy and image quantification}

Fixed or live embryos were mounted with $1.5 \%$ low melting agarose (140727, SERVA) and imaged using a Leica TCS SPE confocal microscope. For quantification of numbers of GFPLc3-positive vesicles, the fixed $4 \mathrm{dpf}$ larvae were imaged by confocal microscopy with a $\times 63$ water immersion objective (NA 1.2) in the pre-defined tail fin region to detect the number of GFP-LC3-positive vesicles (Fig. 3a, b). The numbers of GFP-Lc3 vesicles were measured by Fiji/ImageJ software (Fig. 3a, b) ${ }^{68}$. For quantification of the autophagic response targeted to $\mathrm{Mm}$ clusters (Fig. 3f, g), the fixed $2 \mathrm{dpi}$ larvae were imaged by confocal microscopy with a $\times 40$ water immersion objective (NA 0.8) over the whole caudal hematopoietic tissue (CHT) region. The same approach was used to quantify Mm acidification in the CHT region (Fig. 5b, c). To investigate the intramacrophage or extracellular localization of bacteria, fixed larvae were again imaged over the CHT as described above, after which the total number of Mm clusters and the number of clusters inside macrophages were counted. To assay cell death, images from fixed $2 \mathrm{dpi}$ larvae were acquired as above, and the number of cells positive for TUNEL staining in the CHT region was counted manually.

\section{mRNA preparation and injection}

dram1 or $\operatorname{dram}^{\Delta 19 \mathrm{~N}}$ (negative control) RNA was isolated from wild type or $\operatorname{dram1} 1^{\Delta 19 n / \Delta 19 n}$ embryos using QIAzol lysis reagent (79306, QIAGEN) and purified with the RNeasy MinElute Cleanup kit (74204, QIAGEN). cDNA synthesis was performed using the iScript cDNA synthesis kit (1708891, BIO-RAD). Full-length dram1 cDNA and dram $1^{\Delta 19 \mathrm{~N}} \mathrm{cDNA}$ was obtained by PCR amplification using Phusion High-Fidelity DNA Polymerase (M0530S, New England Biolabs). The following primers were used: Forward: 5'-CTGCGGCGAGATGT TTTGGTT-3'; Reverse: 5'-CAAAAACAGTGGGACATACAGTGAA-3'. dram1 or dram ${ }^{\Delta 19 \mathrm{~N}}$ PCR products were ligated into a ZERO BLUNT TOPO vector (450245, ThermoFisher) and the insert was confirmed by Sanger sequencing (Base Clear, Netherlands). dram1 and dram $1^{\Delta 19 \mathrm{~N}}$ mRNA was generated using the SP6 mMessage mMachine kit (AM1340, Thermo Fisher) and Poly(A) Tailing Kit (AM1350, ThermoFisher); purified using the RNeasy Mini Elute Cleanup kit (74204, QIAGEN) and 50 pg mRNA was microinjected into one-cell stage embryos.

\section{TUNEL assay}

Cell death was examined by Terminal deoxynucleotidyl TUNEL staining with the In Situ Cell Death Detection Kit, TMR red (1256792910, SIGMAALDRICH) in 2dpi fixed embryos. The assay was performed as follows: embryos were fixed in 4\% PFA in PBS solution $\mathrm{O} / \mathrm{N}$ at $4{ }^{\circ} \mathrm{C}$, de-hydrated and re-hydrated in serial dilutions of methanol $(\mathrm{MeOH})(25 \%, 50 \%, 75 \%$, $100 \%, 75 \%, 50 \%, 25 \%)$ and washed in PBS containing $0.1 \%$ Triton-X100. Then, embryos were permeabilized in $10 \mu \mathrm{g} / \mathrm{ml}$ Proteinase $\mathrm{K}$ for $40 \mathrm{~min}$ at $37^{\circ} \mathrm{C}$ followed by a quick rinse in PBS containing $0.1 \%$ Triton X100. TUNEL staining was performed according to kit instructions $\mathrm{O} / \mathrm{N}$ at $37^{\circ} \mathrm{C}$ in the dark. Samples were washed and stored in PBST until imaging by confocal microscopy as described above.

\section{LysoTracker staining and Myeloperoxidase (Mpx) activity assay}

Infected embryos were immersed in egg water with $10 \mu \mathrm{M}$ LysoTracker Red DND-99 (L7528, ThermoFisher) for $1 \mathrm{~h}$. Embryos were washed three times with egg water before imaging. Mpx activity assay was performed with the Leukocyte detection Kit (390A, SIGMA-ALDRICH) for detection of neutrophils as previously described ${ }^{69}$.

\section{Drug treatment}

Embryos were bath treated with Bafilomycin A1 (BafA1) (B1793-10UG, SIGMA-ALDRICH) diluted into egg water at the working concentration of $100 \mathrm{nM}$ for $12 \mathrm{~h}$.

\section{Caspase activity assay \\ Inflammatory caspase activity was assayed with the fluorometric substrate Z-YVAD 7-Amido-4- trifluoromethylcoumarin (Z-YVAD-AFC, Caspase-1}


Substrate IV, Colorimetric, sc-311283, Santa Cruz) as described previously ${ }^{70}$. 35 embryos/group were sonicated and incubated in hypotonic cell lysis buffer (25 mM 4-(2-hydroxyethyl) piperazine-1-ethanesulfonic acid, $5 \mathrm{mM}$ ethylene glycol-bis (2-aminoethyl ether)-N, $\mathrm{N}, \mathrm{N}^{\prime}, \mathrm{N}^{\prime}$-tetraacetic acid, $5 \mathrm{mM}$ dithiothreitol, $\mathrm{pH}$ 7.5) on ice for $15 \mathrm{~min}$. For each reaction, $10 \mu \mathrm{g}$ protein was incubated for $90 \mathrm{~min}$ at $28^{\circ} \mathrm{C}$ with $50 \mu \mathrm{M}$ YVAD-AFC in $50 \mu$ of reaction buffer (0.2\% 3-[(3-cholamidopropyl) dimethylammonio]-1-propanesulfonate (CHAPS), $0.2 \mathrm{M}$ 4-(2-hydroxyethyl) piperazine-1-ethanesulfonic acid, $20 \%$ sucrose, $29 \mathrm{mM}$ dithiothreitol, $\mathrm{pH}$ 7.5). After the incubation, fluorescence was measured in a Tecan M1000 microplate reader at an excitation wavelength of 400 and emission wavelength of $505 \mathrm{~nm}$.

\section{Morpholino injection conditions}

Previously validated caspa and gsdmeb morpholinos $(\mathrm{MO})^{71}$ were purchased from Gene Tools (Gene Tools, USA). MO oligonucleotide sequence: caspa 5'-GCCATGTT TAGCTCAGGGCGCTGAC-3' ${ }^{\prime}$ gsdmeb MO 5'-TCATGCT CATGCTAGTCAGGGAGG- $3^{71}$. MOs were diluted in Milli-Q water with $0.05 \%$ phenol red and $1 \mathrm{~nL}$ of $0.6 \mathrm{mM}$ (caspa MO) or $0.7 \mathrm{mM}$ (gsdmeb) MO was microinjected into the yolk of one-cell stage embryos as previously described (39).

\section{Statistical analyses and data representation}

Power calculations where performed to ensure that sufficient animals were included in the experiments to be able to detect a effect size of at least $20 \%$. For infection experiments, improperly injected animals were excluded from the experiment. No animals were excluded from the analysis after the initial selection. Wild type and mutant animals were randomly distributed over the different experimental conditions and blinding was applied during data acquisition and analysis when possible. Statistical analyses were performed using GraphPad Prism software (Version 5.01; GraphPad). All experimental data (mean \pm SEM) was analyzed using unpaired, two-tailed Mann-Whitney $U$ tests for comparisons between two groups and Kruskal-Wallis one-way analysis of variance with Dunn's multiple comparison methods as a posthoc test for comparisons between more than two groups. (ns, no significant difference; $" p<0.05$; $* p<0.01$; $* * * 0<0.001)$. For segregation of F1 or F3 heterozygous offspring, data were analyzed with a Chi-square test (ns, no significant difference). Continuous measurements are represented as bar graphs. In non-continuous measurements, the data sets from each group are shown in a scatter plot (left) and a boxplot (right). In the scatter plots each data point represents an individual embryo/larva, with the mean indicated by a horizontal line. Boxplots include $50 \%$ of the data points, with a vertical line indicating the $95 \%$ confidence interval and a horizontal line indicating the median.

\section{Acknowledgements}

We thank Daniel Klionsky for sharing the CMV:EGFP-map1/c3b transgenic zebrafish line and Georges Lutfalla for the mpeg 1:mCherryF line. We are grateful to all members of the fish facility team for zebrafish caretaking. We would like to thank Gerda Lamers and Joost Willemse for advice on confocal imaging and image analysis. R.Z. was supported by a grant from the China Scholarship Council (CSC). M.V. and G.F.-C. were funded by European Marie Curie fellowships (H2020-MSCA-IF-2014-655424 and H2020-COFUND-2015-FP707404), V.T. was a Marie Curie fellow in the Initial Training Network FishForPharma (PITN-GA-2011-289209), and M.v.d.V. was supported by the Netherlands Technology Foundation TTW (project 13259).

\section{Conflict of interest}

The authors declare that they have no conflict of interest.

\section{Publisher's note}

Springer Nature remains neutral with regard to jurisdictional claims in published maps and institutional affiliations.

Supplementary Information accompanies this paper at (https://doi.org/ 10.1038/s41419-020-2477-1).

Received: 13 November 2019 Revised: 7 April 2020 Accepted: 8 April 2020 Published online: 24 April 2020

\section{References}

1. Ohsumi, Y. Yoshinori Ohsumi: autophagy from beginning to end. Interview by Caitlin Sedwick. J. Cell Biol. 197, 164-165 (2012).

2. Levine, B., Mizushima, N. \& Virgin, H. W. Autophagy in immunity and inflammation. Nature 469, 323-335 (2011).

3. Deretic, V., Saitoh, T. \& Akira, S. Autophagy in infection, inflammation and immunity. Nat. Rev. Immunol. 13, 722 (2013).

4. Wallis, R. S. \& Hafner, R. Advancing host-directed therapy for tuberculosis. Nat. Rev. Immunol. 15, 255-263 (2015).

5. Paik, S., Kim, J. K., Chung, C. \& Jo, E. K. Autophagy: a new strategy for hostdirected therapy of tuberculosis. Virulence 1-12, https://doi.org/10.1080/ 21505594.2018.1536598 (2018).

6. van der Vaart, M. et al. The DNA damage-regulated autophagy modulator DRAM1 links mycobacterial recognition via TLR-MYD88 to autophagic defense [corrected]. Cell Host Microbe 15, 753-767 (2014).

7. Gutierrez, M. G. et al. Autophagy is a defense mechanism inhibiting BCG and Mycobacterium tuberculosis survival in infected macrophages. Cell 119, 753-766 (2004).

8. Watson, R. O., Manzanillo, P. S. \& Cox, J. S. Extracellular M. tuberculosis DNA targets bacteria for autophagy by activating the host DNA-sensing pathway. Cell 150, 803-815 (2012).

9. Krakauer, T. Inflammasomes, autophagy, and cell death: the trinity of innate host defense against intracellular bacteria. Mediators Inflamm. 2019, 2471215 (2019).

10. López-Jiménez, A. T. et al. The ESCRT and autophagy machineries cooperate to repair ESX-1-dependent damage at the Mycobacterium-containing vacuole but have opposite impact on containing the infection. PLOS Pathog. 14, e1007501 (2019).

11. Crighton, D. et al. DRAM, a p53-induced modulator of autophagy, is critical for apoptosis. Cell 126, 121-134 (2006).

12. Guan, J. J. et al. DRAM1 regulates apoptosis through increasing protein levels and lysosomal localization of BAX. Cell Death Dis. 6, e1624 (2015).

13. Humbert, M., Mueller, C., Fey, M. F. \& Tschan, M. P. Inhibition of damageregulated autophagy modulator-1 (DRAM-1) impairs neutrophil differentiation of NB4 APL cells. Leuk. Res. 36, 1552-1556 (2012).

14. Laforge, M. et al. DRAM triggers lysosomal membrane permeabilization and cell death in CD4(+) T cells infected with HIV. PLoS Pathog. 9, e1003328 (2013).

15. Mrschtik, M. \& Ryan, K. M. Another DRAM involved in autophagy and cell death. Autophagy 12, 603-605 (2016).

16. Wu, X. et al. Increased expression of DRAM1 confers myocardial protection against ischemia via restoring autophagy flux. J. Mol. Cell. Cardiol. 124, 70-82 (2018).

17. Houben, D. et al. ESX-1-mediated translocation to the cytosol controls virulence of mycobacteria. Cell. Microbiol. 14, 1287-1298 (2012). 
18. Ponpuak, M. et al. Delivery of cytosolic components by autophagic adaptor protein p62 endows autophagosomes with unique antimicrobial properties. Immunity 32, 329-341 (2010).

19. Zhang, R. et al. The selective autophagy receptors Optineurin and p62 are both required for zebrafish host resistance to mycobacterial infection. PLoS Pathog. 15, e1007329 (2019).

20. Ramakrishnan, L. Revisiting the role of the granuloma in tuberculosis. Nat. Rev. Immunol. 12, 352-366 (2012).

21. Srinivasan, L., Ahlbrand, S. \& Briken, V. Interaction of Mycobacterium tuberculosis with host cell death pathways. Cold Spring Harb. Perspect. Med. 4, https://doi. org/10.1101/cshperspect.a022459 (2014).

22. Gan, H. et al. Mycobacterium tuberculosis blocks crosslinking of annexin-1 and apoptotic envelope formation on infected macrophages to maintain virulence. Nat. Immunol. 9, 1189-1197 (2008).

23. Behar, S. M. et al. Apoptosis is an innate defense function of macrophages against Mycobacterium tuberculosis. Mucosal Immunol. 4, 279-287 (2011).

24. Roca, F. J. \& Ramakrishnan, L. TNF dually mediates resistance and susceptibility to mycobacteria via mitochondrial reactive oxygen species. Cell 153, 521-534 (2013).

25. Stutz, M. D., Clark, M. P., Doerflinger, M. \& Pellegrini, M. Mycobacterium tuberculosis: rewiring host cell signaling to promote infection. J. Leukoc. Biol. 103 259-268 (2018)

26. He, C., Bartholomew, C. R., Zhou, W. \& Klionsky, D. J. Assaying autophagic activity in transgenic GFP-LC3 and GFP-Gabarap zebrafish embryos. Autophagy 5, 520-526 (2009)

27. Bernut, A. et al. Mycobacterium abscessus cording prevents phagocytosis and promotes abscess formation. Proc. Natl Acad. Sci. USA 111, E943-E952 (2014).

28. Benard, E. L., Rougeot, J., Racz, P. I., Spaink, H. P. \& Meijer, A. H. Transcriptomic approaches in the Zebrafish model for tuberculosis-insights into host- and pathogen-specific determinants of the innate immune response. Adv. Genet. 95, 217-251 (2016)

29. de Carvalho, L. P. et al. Metabolomics of Mycobacterium tuberculosis reveals compartmentalized co-catabolism of carbon substrates. Chem. Biol. 17 1122-1131 (2010).

30. Gatica, D., Lahiri, V. \& Klionsky, D. J. Cargo recognition and degradation by selective autophagy. Nat. Cell Biol. 20, 233-242 (2018).

31. Cambier, C. J., O'Leary, S. M., O'Sullivan, M. P., Keane, J. \& Ramakrishnan, L. Phenolic glycolipid facilitates mycobacterial escape from microbicidal tissueresident macrophages. Immunity 47, 552-565 e554 (2017).

32. Miao, E. A., Rajan, J. V. \& Aderem, A. Caspase-1-induced pyroptotic cell death Immunol. Rev. 243, 206-214 (2011)

33. Kyrylkova, K., Kyryachenko, S., Leid, M. \& Kioussi, C. Detection of apoptosis by TUNEL assay. Methods Mol. Biol. 887, 41-47 (2012).

34. Galluzzi, L. et al. Molecular mechanisms of cell death: recommendations of the Nomenclature Committee on Cell Death 2018. Cell Death Differ. 25, 486-541 (2018).

35. Porter, A. G. \& Janicke, R. U. Emerging roles of caspase-3 in apoptosis. Cell Death Differ. 6, 99-104 (1999).

36. Shi, J., Gao, W. \& Shao, F. Pyroptosis: gasdermin-mediated programmed necrotic cell death. Trends Biochem. Sci. 42, 245-254 (2017).

37. Shi, J. et al. Cleavage of GSDMD by inflammatory caspases determines pyroptotic cell death. Nature 526, 660 (2015).

38. Kayagaki, N. et al. Caspase-11 cleaves gasdermin D for non-canonical inflammasome signalling. Nature 526, 666 (2015).

39. Varela, M., van der Vaart, M., Groenewoud, A. \& Meijer, A. H. Extracellular mycobacterial DNA triggers Caspase-11-dependent pyroptosis of infected macrophages and drives disease progression. Preprint at https:/doi.org/ 10.1101/514125 (2019)

40. Forn-Cuni, G., Meijer, A. H. \& Varela, M. Zebrafish in inflammasome research. Cells 8, https://doi.org/10.3390/cells8080901 (2019).

41. Perera, R. M. \& Zoncu, R. The lysosome as a regulatory hub. Annu. Rev. Cell Dev. Biol. 32, 223-253 (2016)

42. O'Neill, L. A. \& Pearce, E. J. Immunometabolism governs dendritic cell and macrophage function. J. Exp. Med. 213, 15-23 (2016).

43. Quinlivan, V. H. \& Farber, S. A. Lipid uptake, metabolism, and transport in the larval zebrafish. Front Endocrinol. 8, 319 (2017).

44. Nagata, M. et al. Dram1 regulates DNA damage-induced alternative autophagy. Cell Stress 2, 55-65 (2018).

45. Galavotti, S. et al. The autophagy-associated factors DRAM1 and p62 regulate cell migration and invasion in glioblastoma stem cells. Oncogene $\mathbf{3 2}$, 699-712 (2013).
46. Zhang, X. D., Qi, L., Wu, J. C. \& Qin, Z. H. DRAM1 regulates autophagy flux through lysosomes. PLOS ONE 8, e63245 (2013).

47. Davis, J. M. \& Ramakrishnan, L. The role of the granuloma in expansion and dissemination of early tuberculous infection. Cell 136, 37-49 (2009).

48. Cookson, B. \& Brennan, M. Pro-inflammatory programmed cell death. Trends Microbiol. 9, 113-114 (2001)

49. Bergsbaken, T., Fink, S. L. \& Cookson, B. T. Pyroptosis: host cell death and inflammation. Nat. Rev. Microbiol. 7, 99-109 (2009).

50. Danelishvili, L., Everman, J. L., McNamara, M. J. \& Bermudez, L. E. Inhibition of the plasma-membrane-associated serine protease cathepsin $\mathrm{G}$ by $\mathrm{Myco}_{\mathrm{c}}$ bacterium tuberculosis Rv3364c suppresses caspase-1 and pyroptosis in macrophages. Front. Microbiol. 2, 281 (2011)

51. Mahamed, D. et al. Intracellular growth of Mycobacterium tuberculosis after macrophage cell death leads to serial killing of host cells. Elife $\mathbf{6}$, https://doi. org/10.7554/eLife.22028 (2017).

52. Labun, K., Montague, T. G., Gagnon, J. A., Thyme, S. B. \& Valen, E. CHOPCHOP V2: a web tool for the next generation of CRISPR genome engineering. Nucleic Acids Res. 44, W272-W276 (2016).

53. RStudio: Integrated Development for R. (RStudio, Inc., Boston, MA, 2015).

54. R: A language and environment for statistical computing (The R Foundation for Statistical Computing, Vienna, 2011).

55. Robinson, M. D., McCarthy, D. J. \& Smyth, G. K. edgeR: a bioconductor package for differential expression analysis of digital gene expression data. Bioinformatics 26, 139-140 (2010)

56. Love, M. I., Huber, W. \& Anders, S. Moderated estimation of fold change and dispersion for RNA-seq data with DESeq2. Genome Biol. 15, 550 (2014).

57. Chen, H. VennDiagram: Generate High-Resolution Venn and Euler Plots. R package version 1.6 .20 (2018).

58. Subramanian, A., Tamayo, P., Mootha, V. K., Mukherjee, S. \& Mesirov, J. P. Gene set enrichment analysis: a knowledge-based approach for interpreting genome-wide expression profiles. Proc. Natl Acad. Sci. USA 102, 15545-15550 (2005).

59. Young, M. D., Wakefield, M. J. Smyth, G. K. \& Oshlack, A. Gene ontology analysis for RNA-seq: accounting for selection bias. Genome Biol. 11, R14 (2010).

60. Rainer, J., Gatto, L. \& Weichenberger, C. X. ensembldb: an R package to create and use Ensembl-based annotation resources. Bioinform Oxf Engl $\mathbf{3 5}$ 3151-3153 (2019)

61. Durinck, S., Spellman, P. T., Birney, E. \& Huber, W. Mapping identifiers for the integration of genomic datasets with the R/Bioconductor package biomaRt Nat. Protoc. 4, 1184-1191 (2009).

62. Ritchie, M. E. et al. limma powers differential expression analyses for RNA-sequencing and microarray studies. Nucleic Acids Res. 43, e47 (2015).

63. Luo, W. \& Brouwer, C. Pathview: an R/Bioconductor package for pathway-based data integration and visualization. Bioinformatics 29, 1830-1831 (2013)

64. Takaki, K., Davis, J. M., Winglee, K. \& Ramakrishnan, L. Evaluation of the pathogenesis and treatment of Mycobacterium marinum infection in zebrafish. Nat. Protoc. 8, 1114-1124 (2013).

65. van der Sar, A. M. et al. Mycobacterium marinum strains can be divided into two distinct types based on genetic diversity and virulence. Infect. Immun. 72 6306-6312 (2004)

66. Benard, E. L. et al. Infection of zebrafish embryos with intracellular bacterial pathogens. J. Vis. Exp. https://doi.org/10.3791/3781 (2012).

67. Stoop, E. J. M. et al. Zebrafish embryo screen for mycobacterial genes involved in the initiation of granuloma formation reveals a newly identified ESX-1 component. Dis. Models Mech. 4, 526-536 (2011).

68. Schindelin, J. et al. Fiji: an open-source platform for biological-image analysis Nat. Methods 9, 676-682 (2012).

69. Cui, C. et al. Infectious disease modeling and innate immune function in zebrafish embryos. Methods Cell Biol. 105, 273-308 (2011).

70. Tyrkalska, S. D. et al. Neutrophils mediate Salmonella Typhimurium clearance through the GBP4 inflammasome-dependent production of prostaglandins. Nat. Commun. 7, 12077 (2016)

71. Masumoto, J. et al. Caspy, a zebrafish caspase, activated by ASC oligomerization is required for pharyngeal arch development. J. Biol. Chem. 278, 4268-4276 (2003) 